\section{Influenza Vaccination}

\section{A Call for a Multiple Intervention Approach}

\author{
Donna M. Pierrynowski Gallant, $\mathrm{PhD}, \mathrm{RN}^{1}$ \\ Mary Ann Murray, PhD (candidate), $\mathrm{RN}^{2}$ \\ Shelly McNeil, MD, FRCPC ${ }^{3}$
}

\section{ABSTRACT}

Influenza seriously impacts the health and well-being of individuals and communities and has significant implications for the health care system. Despite known benefits of influenza vaccination, inoculation rates among health care providers remain low. Close proximity to patients, the potential to act as an infection vector and their role in patient education has focussed attention on how health care professionals make personal vaccination decisions. This commentary explores the inherent complexity of vaccination decision-making and offers suggestions for a multiple intervention approach to address health care providers' vaccination uptake needs. Directions for future research are also discussed.

MeSH terms: Influenza; health personnel; intervention studies; decision making

\section{RÉSUMÉ}

La grippe porte gravement atteinte à la santé et au bien-être des personnes et des collectivités, et elle a des répercussions importantes sur le système de soins de santé. Malgré les avantages connus du vaccin antigrippal, les taux d'inoculation des dispensateurs de soins de santé demeurent faibles. Or, leurs liens étroits avec les patients, la possibilité qu'ils propagent les infections et leur rôle dans la sensibilisation des patients attirent l'attention sur la façon dont les professionnels de la santé prennent individuellement la décision de se faire vacciner ou non. Ce commentaire traite de la complexité intrinsèque du processus décisionnel en matière de vaccination et propose une approche axée sur les interventions multiples pour convaincre les dispensateurs de soins de santé d'accepter de se faire vacciner. Il est aussi question des pistes de recherche à explorer.
1. Assistant Professor, School of Nursing, Saint Francis Xavier University, Antigonish, NS

2. Doctoral student, School of Nursing, University of Ottawa, Ottawa, ON

3. Assistant Professor of Medicine, Division of Infectious Diseases, Dalhousie University, Halifax, NS Correspondence and reprint requests: Donna M. Pierrynowski Gallant, School of Nursing, Saint Francis Xavier University, Box 5000, Antigonish, NS B2G 2W5, Tel: 902-867-3392, Fax: 902-8672322,E-mail: dgallant@stfx.ca
I nfluenza negatively impacts the health of individuals and communities and the functioning of the health care system, ${ }^{1}$ and is a major contributor to mortality, morbidity, and lost productivity. In Canada, between one in four to one in ten people contract influenza and 500-1500 people die of influenza annually. ${ }^{2}$ Although the influenza vaccine is both clinically and economically effective, vaccination uptake rates remain sub-optimal in all groups for whom the vaccine is recommended. In Canada, 70-91\% of longterm-care residents and $20-40 \%$ of adults and children with medical conditions typically receive the vaccine. ${ }^{3}$ Recent studies also reveal that only $26-61 \%$ of health care workers become vaccinated. ${ }^{4-7}$ Sub-optimal vaccination rates among health care providers are of particular concern as unvaccinated personnel can act as transmission vectors to high-risk patients. Health care workers also play an important role in the education of patients and their families about influenza vaccination and may be viewed as role models by their patients. The purpose of this commentary is to summarize the parameters regarding decision making in the context of health worker vaccination. Our goal is to invoke a collective commitment to work at multiple levels through multiple interventions to address health care worker influenza vaccination as an important health care promotion agenda.

Although low rates of influenza vaccine uptake have been attributed to the failure of the health care system to offer the vaccine and to provider's vaccination refusal, ${ }^{3}$ little is currently known about the influen$\mathrm{za}$ vaccination decision-making process engaged in by health care providers annually. Lack of a clear understanding of the type and ranking of decision determinants they consider when making a decision regarding influenza vaccination may limit the efficacy of decision support interventions provided by other health care professionals and policy-makers. Further research is required to inform the development of responsive, effective interventions. Concurrently, we can move forward to address some particular issue areas.

Decision theory suggests that a complex interplay between cognitive, emotional and social interactions and contextual factors among individuals, health care providers, peer groups and organizational 
infrastructures impacts health decisionmaking. ${ }^{8}$ Specifically, the decision to accept or reject the influenza vaccine is often shaped by the combined impact of social expectations and previous experience with influenza. ${ }^{9}$ Therefore the reciprocal connections among behaviour, environment, and policy related to providers' decision to accept or reject influenza vaccination and the impact of providers' personal choice on client decision-making must be acknowledged when planning, promoting and implementing influenza vaccination programs and facilitative structures. ${ }^{10}$

The need to address multiple individual and environmental factors such as providers' personal knowledge, social reasoning and norms, approaches to the patient teaching process, perceived barriers and facilitators to immunization access, and the availability of supportive organizational infrastructures requires a multiple intervention approach. ${ }^{10}$ This socio-ecological approach offers an expansive framework for understanding and modifying the range of social and environmental factors that shape providers' decision-making.

A single strategy is insufficient to improve vaccination uptake rates. Combined intervention programs are associated with better vaccination coverage compared to single component interventions that have minimal effectiveness. ${ }^{6,11}$ Various reciprocating elements between individual variables in conjunction with interpersonal relationships between group members and within the larger social system, ${ }^{12}$ need to be considered when developing a comprehensive influenza program. A multiple intervention strategy, designed to enhance decision support interventions and vaccination availability infrastructures at multiple levels, provides a practical platform to launch a comprehensive vaccination uptake initiative and a program of research. ${ }^{10} \mathrm{~A}$ multiple intervention design directed at multiple levels is recommended to optimize the probability of influencing change and sustaining outcomes. Target areas should include educational settings, clinical practice environments, organizational structures, professional associations and the broader socio-political arena. ${ }^{13}$

Employers of health care providers can develop strategies that assist providers in mastering skills necessary to allow them to provide decision support and patient edu- cation at the direct point of care. Provider "champions" across organizations have the potential to exponentially spread successful initiatives and ideas related to optimizing decision support mechanisms for health care professionals across and between institution boundaries. ${ }^{14}$ Administrators can act as important innovation facilitators and systems brokers. For example, a regional consortium of long-term-care facilities may find it useful to pool efforts to develop a vaccination education and outreach program for employees within their various settings. Collectively, these groups can create synergy and can crystallize a "tipping point" to influence attitudes and behaviours related to a good practice approach to health care provider's vaccination uptake. ${ }^{15}$

As self-regulated professionals, providers such as nurses and physicians have an obligation to promote the best outcomes for patients and their families. In addition, the National Advisory Committee on Immunization (NACI) "considers the provision of influenza vaccination for health care workers involved in direct patient care to be an essential component of the standard of care for influenza prevention for the protection of their patients. Health care workers involved in direct patient care should consider it their responsibility to provide the highest standard of care, which includes undergoing annual influenza vaccination. In the absence of contraindications, refusal of health care workers who are involved in direct patient care to be immunized against influenza implies failure in their duty of care to their patients." 3 Exploring the boundary lands between professional standards, expert opinion and providers' personal health choices may provide new insights and areas ripe for research. Issue clarification and consensus building may then occur at both the individual and collective level. In addition, regulatory bodies and professional organizations could collaborate to develop, implement, and evaluate decision support interventions for those who are ambivalent about the influenza vaccine.

Within the current environment of risk reduction, patients, health care professionals and organizational decision-makers are challenged to understand and address health care providers' vaccination needs. Policy-makers, health promotion leaders and organizational decision-makers can develop and use innovative communications media to reach health care providers and students. ${ }^{13}$ Policies that endorse the use of vaccination uptake need to be practical, visible, accessible, evidence-based and equitably enforced. Attention to environmental barriers and supportive infrastructures must be addressed in policies outlining influenza vaccination expectations and outbreak response.

An emphasis on quick fixes to complex problems such as influenza vaccine uptake is limiting. Health care is a partnership of individuals, care providers and organizational structures. A multiple intervention approach represents people working together to achieve practical outcomes. Understanding how to separate and to share the responsibility for minimizing the impact of influenza infection at the individual, community and population level requires consideration of the relationship between different providers, their diversity, their shared environment, organizational infrastructures and the socio-political context that act as facilitators or barriers to influenza vaccination uptake. ${ }^{10} \mathrm{~A}$ multiple intervention approach provides a practical, innovative way to guide a comprehensive program to address health care provider vaccination uptake needs and to construct a much-needed research agenda. Research inquiry into areas such as what is the best combination, dose and timing of intervention strategies may provide insights for constructing multiple intervention programs. Other areas of research may focus on the impact of interdisciplinary education and how to best reach out to providers as members of individual disciplines or as members of interdisciplinary teams.

\section{REFERENCES}

1. World Health Organization, editor. Influenza (fact sheet no. 211). Geneva, Switzerland: WHO, 2003. Available online at: http://www.who.int/ mediacentre/factsheets/fs $211 /$ en/ (Accessed September 24, 2004).

2. Health Canada, Population and Public Health Branch. Influenza information. Available online at: http://www.hc-sc.gc.ca/pphb-dgspsp/publicat/info/infflue.html (Accessed August 24, 2002).

3. National Advisory Committee on Immunization. Statement on influenza vaccination for the 2004 2005 season; 2004. Can Commun Dis Rep 30(ACS-3), 1-13.

4. DeWals P, Carbonneau M, Payette H. Influenza and pneumococcal vaccination in long term care facilities in two regions of Quebec. Can J Infect Dis 1996;7:296-300.

5. Stevenson C, McArthur M, Naus M, Abraham E, McGeer A. Prevention of influenza and pneu- 
mococcal pneumonia in Canadian long term facilities: How are we doing? CMAJ 2001;164(10):1413-19.

6. Russell M. Influenza vaccination in Alberta long term care facilities. CMAJ 2001;164(10):1423-27.

7. Nichol K, Hauge M. Influenza vaccination of health care workers. Infect Control Epidemiol 1997;18:189-94.

8. O'Connor A, Jacobsen M (Eds.). Decisional conflict: Assessing and supporting patients experiencing uncertainty about choices affecting their health [monograph on the internet]. The Ottawa Health Decision Center at the Ottawa Health Research Institute, 2004. Available online at: decisionaid.ohri.ca/docs/Reading_DC_0204.pdf (Accessed June 14, 2004).
9. Pierrynowski Gallant D, Robinson Vollman A Influenza vaccine choices. Can Nurse 2004;100(2):16-22.

10. Edwards N, Mills J, Kothari A. Multiple intervention research programs in community health. Can J Nurs Res 2004;36(1):40-54.

11. Shefer A, Briss P, Rodewald L, Bernier R, Strikas $\mathrm{R}$, Yusaf $\mathrm{H}$. Improving immunization coverage rates: An evidence based review of the literature. Epidemiol Rev 1999;21(1):96-142.

12. Sallis J, Owen N. Ecological models. In: Glanz K, Lewis F, Rimer R (Eds.), Health Behaviors and Health Education: Theory, Research and Practice, $2^{\text {nd }}$ ed. San Francisco, CA: Jossey Bass, 1997;403-24.

13. Pierrynowski Gallant D. Influenza vaccination: A personal decision. Doctoral dissertation (unpub-

\section{Book Reviews/Recension}

\section{Fatal Indifference: The G8, Africa and Global Health}

Ron Labonte and Ted Schrecker, with David Sanders and Wilma Meeus. Ottawa, Ontario: The University of Cape Town Press and International Development Research Centre, 2004; 378 pp.

Fatal Indifference presents a "report card" for critical monitoring of the health implications of G8 country actions, or "(in)actions" on health. The message one takes from this book is that G8 commitments to health and development are often broken, and that in many cases the commitments are not sufficient to address the enormity of the problems faced by poor countries. The book provides evidence that within the context of 'globalization' or 'liberalization', there has been an evident slowdown in development progress. The analysis is of key texts of commitments made at the G8 summits in Cologne (1999), Okinawa (2000) and Genoa (2001).

The book begins with an introduction clearly describing the measures used, the analytic framework, and the limitations of the analysis. The first chapter outlines the nature of globalization and its relationship with health and development. It is here that the authors explain their perspective of the G8 countries as the emerging centre of global governance, backed by economic influence. The subsequent seven chapters each examine a separate area of G8 policy commitments and their relationship to health (e.g., macroeconomic policy, structural adjustment and debt relief; official development assistance; trade and market access).

The book will be of interest to a wide array of scholars/activists working in global health and the determinants of health. It successfully draws the linkages between the various determinants of health (health, education, nutrition and economics) and the effect of policies and programs on the determinants. While it would likely be understandable to even the novice reader because it provides clear definitions, descriptions and a framework for the analysis, the evidence presented is a bit dense for the uninitiated. The book is well written and organized and makes effective use of specific examples in boxes that fit smoothly within the overall text of each chapter. The presentation of evidence in tables and graphs is clear and helpful to understanding of the text. There is an extensive list of references provided, and appendices provide more detailed information.

$$
\begin{aligned}
& \text { Lory Laing, PhD } \\
& \text { Professor, Public Health Sciences } \\
& \text { Faculty of Medicine and Dentistry } \\
& \text { University of Alberta }
\end{aligned}
$$

\section{The Combined Approach Matrix: A Priority Setting Tool for Health Research} A. Ghaffar, A. de Francisco, \& S. Matlin (Eds.). Global Forum for Health Research. 2004; 68 pp.

In 1990, the Commission on Health Research for Development stated that less than 10\% of global research funds, from public and private sources, are directed to $90 \%$ of the world's health problems. In response, the basic thesis of The Combined Approach Matrix is that changing the priorities that determine how existing health research funds are used can rectify this 10/90 gap. The authors discuss the challenges associated with priority setting, both in terms of the processes involved and tools used. They present a tool, the Combined Approach Matrix (CAM), developed by the Global Forum on Health Research, to assist institutions (at the national, regional and global levels) in setting priorities in health research. The matrix is composed of previously developed criteria and prin- lished manuscript). University of Calgary, Calgary, AB, 2005.

14. Nafziger D, Herwaldt L. Attitudes of internal medicine residents regarding influenza vaccination. Infection Control and Hospital Epidemiology 1994;15(1):32-35.

15. Gladwell M. The tipping point: How little things can make a big difference. Boston, MA: Little Brown \& Co., 2002.

Received: October 5, 2004

Revisions requested: December 15, 2004

Revised ms: July 8, 2005

Accepted: August 18, 2005

ciples that are linked to stakeholders and determinants of population health status. One axis of the matrix provides a five-step methodology (linking disease burden, with determinants, cost-effectiveness and financial flows) while the other axis recognizes the non-biomedical influencing factors on health (individual and community behaviour, governmental/macroeconomic policies, agriculture, transportation, etc.)

The strength of this publication is in its application of the CAM model to different jurisdictions (global, national), diseases, risk factors and vulnerable target groups - thus showing how it can combine a diverse range of health-related factors to set priorities in research. Practical challenges are identified as data collection (e.g., not available) and/or transforming data into meaningful information (e.g., consistent format). The document also provides a brief understanding of how the CAM can be tailored to accommodate other challenges (e.g., using the CAM to highlight the most important gaps in information needed for decision-making). A limitation of the publication is that its discussion remains at a fairly conceptual level. Even the applications include only the fundamentals of the Matrix at work. Further, while the importance of an institution's underlying values to priority setting is acknowledged, they are not openly included in the matrix. Therefore, the manual provides insufficient concrete detail for more than a broad understanding of the tool. Nevertheless, this publication can serve as a useful tool to build awareness (among policy-makers, academics and providers) and in identifying essential factors for consideration to effect real change in priority setting with respect to health research.

Jean Hughes, $R N, P h D$

School of Nursing

Dalhousie University

and Department of Psychiatry

IWK Health Centre for Women,

Children and Families 